\title{
AVANÇOS E DESAFIOS DA ENFERMAGEM EM ACUPUNTURA EM SANTA CATARINA NO PERÍODO DE 1997 A 2015
}

\section{ADVANCES AND CHALLENGES OF NURSING PRACTICES IN ACUPUNCTURE IN SANTA CATARINA FROM 1997 TO 2015}

\section{AVANCES Y DESAFÍOS DE LA ENFERMERÍA EN ACUPUNTURA EN SANTA CATARINA DE 1997 A 2015}

Ana Paula Senna Bousfield ${ }^{1}$ Maria Itayra Padilha ${ }^{2}$

\section{RESUMO}

Objetivo: Conhecer os avanços na atuação das enfermeiras, na prática da acupuntura, em Santa Catarina, no período de 1997 a 2015. Método: Qualitativo com abordagem Histórico-Social, por meio da história oral temática, fundamentado na sociologia das profissões de Eliot Freidson. A coleta de dados ocorreu, no período de março a maio de 2018. Após análise temática, apontaram-se 2 categorias: Desafios e avanços profissionais e As boas práticas aplicadas na Acupuntura. Resultados: As insatisfações com as entidades de interesse público estão presentes no cotidiano da classe profissional, compondo o cenário dos desafios profissionais. Dentre os avanços identificados, destaca-se que atualmente a acupuntura é realizada apenas por enfermeira especialista na área, seja em consultórios, como em unidade básica de saúde. As boas práticas aplicadas, durante o atendimento da enfermeira acupunturista garantem a qualidade, destaque e a compatibilidade das ações com os regulamentos técnicos. Conclusão: A sociedade brasileira reconhece que as ações desse profissional requerem conhecimento, pesquisa e habilidades para ser exercida. A maior conquista, se relaciona ao encontro com o outro no ato de cuidar.

Descritores: Terapias Complementares; Enfermagem; História da Enfermagem; Ocupações em Saúde.

\section{ABSTRACT}

Objective: To know the advances in the performance of nurses in the practice of acupuncture in Santa Catarina, from 1997 to 2015. Method: Qualitative with Historical-Social approach, through thematic oral history, based on the sociology of the professions by Eliot Freidson. Data collection took place from March to May 2018. After thematic analysis, two categories were reached: Challenges and professional advances; and good practices applied in acupuncture. Results: Dissatisfaction with public entities is present in the daily life of the professionals, creating a scenario of professional challenges. Among the advances identified, it is noteworthy that only specialist nurses in the area practice acupuncture today, whether in private practices or at primary health care services. The good practices applied by the acupuncturist nurse at care guarantee the quality, prominence and compatibility of the actions with the technical regulations. Conclusion: The Brazilian society recognizes that the actions of this professional require knowledge, research and skill. The greatest achievement is related to meeting the other in the act of caring.

Descriptors: Complementary Therapies; Nursing; History of Nursing; Health Occupations.

\section{RESUMEN}

Objetivo: Conocer los avances en el desempeño de las enfermeras en la práctica de la acupuntura en Santa Catarina, de 1997 a 2015. Método: Cualitativo con enfoque histórico-social, a través de la historia oral temática, basada en la sociología de las profesiones de Eliot Freidson. La recopilación de datos tuvo lugar de marzo a mayo de 2018. Después del análisis temático, se definieron dos categorías: desafíos y avances profesionales y; Las buenas prácticas aplicadas en la acupuntura. Resultados: Las insatisfacciones con las entidades de interés público están presentes en la vida diaria de la clase profesional, haciendo parte del escenario de los desafíos profesionales. Entre todos los avances enumerados, se destaca que, hoy día, la acupuntura es realizada solamente por enfermera especialista en el area, sea en consultorios o en unidades básicas de salud. Las buenas prácticas aplicadas durante el cuidado de la enfermera acupunturista garantizan la calidad, la prominencia y la compatibilidad de las acciones con los reglamentos técnicos. Conclusión: La sociedad brasileña reconoce que las acciones de este profesional requieren conocimiento, investigación y habilidades para ser ejercitadas. El mayor logro está relacionado con conocer al otro en el acto de cuidar.

Descriptores: Terapias Complementarias; Enfermería; Historia de la Enfermería; Empleos en Salud.

${ }^{1}$ Enfermeira. Mestre em Enfermagem pela Universidade Federal de Santa Catarina - UFSC. Especialista em Acupuntura pelo Instituto Brasileiro de Terapias e Ensino, Curitiba/Paraná.. ${ }^{2}$ Enfermeira. Doutora em Enfermagem pela EEAN/UFRJ. Titular aposentada da UFSC.

Como citar este artigo:

Bousfield APS. Padilha MI. Avanços e desafios da enfermagem em acupuntura em Santa Catarina no período de 1997 a 2015. Revista de Enfermagem do Centro-Oeste Mineiro. 2020;10:e3666. [Access___]; Available in:___. DOI: http://doi.org/10.19175/recom.v10i0.3666 


\section{INTRODUÇÃO}

A palavra cuidado deriva da palavra latina cogitatus, que expressa meditado, pensado e refletido. Recebe os significados de: atenção especial, inquietação, preocupação, zelo, desvelo que se dedica a alguém ou algo, objeto ou pessoa desse desvelo, encargo, incumbência, responsabilidade, lida, trabalho, ocupação. Os sentidos atribuídos falam da sua dimensão social, implícita na interação entre sujeitos, numa relação de ajuda. Porém, há muitas décadas, o entendimento de cuidado ganhou maior reconhecimento com as ações profissionais na atenção à saúde ${ }^{(1)}$.

$\mathrm{O}$ ato de cuidar e ser cuidado faz parte do ser humano, assim sendo importa clarificar quais os significados específicos de que esse conceito se reveste no âmbito disciplinar de enfermagem. O cuidar, conceito central em enfermagem, influencia a teoria, a investigação, a prática e o ensino. Múltiplas teorias de enfermagem desenvolvem-se em torno desse conceito ${ }^{(2)}$.

Para a profissão de enfermagem, o cuidar influencia a teoria, a investigação, a prática e o ensino. Numerosas teorias de enfermagem foram criadas em torno do cuidar. As enfermeiras são ensinadas a cuidar com qualidade para uma prática assistencial baseada em evidências, necessariamente, resultantes de processos de investigação $^{(2)}$. $O$ cuidado integrado e fundamentado por um corpo de conhecimentos próprios propicia aos profissionais, a realização de práticas que se complementam. Esse cuidado pode ser multiprofissional e interdisciplinar, integrando a acupuntura advinda das terapias complementares.

A Acupuntura é uma técnica de intervenção terapêutica da Medicina Tradicional Chinesa, que se fundamenta na primazia da energia entre a matéria, a pessoa doente e a doença, orientando-se nas características de pessoas com determinados padrões físicos, estruturais, psicológicos e de comportamento ${ }^{(3)}$.

As enfermeiras oferecem uma contribuição singular para a inserção da acupuntura e de outras práticas complementares onde atuam, e o Conselho Federal de Enfermagem (COFEN), em 1997, por meio da Resolução n. 197 reconheceu a acupuntura também como especialidade da enfermeira ${ }^{(4)}$. Em 2008, a atividade de acupuntura foi regulamentada como especialidade, por meio da Resolução COFEN no. $326 / 2008^{(5)}$.
Entendemos que, no Brasil, a acupuntura realizada por enfermeiras ainda está em processo de ampliação, apesar de estar regulamentada. Em 2009, foi criada a Associação Brasileira de Enfermeiros Acupunturistas e Enfermeiros de Práticas Integrativas - ABENAH, pelo Enfermeiro especialista em acupuntura, Daniel Ramos Olcerenko, seu primeiro presidente. A ABENAH tem como missão o compromisso social com a melhoria da qualidade de vida dos brasileiros, dando a oportunidade de atendimento holístico aos que procurarem essa categoria e também de assegurar a esses profissionais o caminho mais assertivo e completo de ferramentas para o atendimento e para a docência, tendo o respaldo institucional dessa associação. A associação possui hoje um ambulatório voluntário junto à Faculdade de Enfermagem da UFG ${ }^{(6)}$.

A implantação de Práticas Integrativas e Complementares no Sistema Público de Saúde (PICS) (SUS) tem sido amplamente discutida nacional e internacionalmente. No Brasil, a Política Nacional de Práticas Integrativas e Complementares já implantou a acupuntura no SUS há cerca de $40 \operatorname{anos}^{(7)}$.

Entre as terapias complementares das PICS, destaca-se a acupuntura, uma técnica milenar da Medicina Tradicional Chinesa, que tem a função de diagnosticar doenças e promover a cura a partir do estímulo da autocura do corpo. A acupuntura teve origem há mais de 5.000 anos sendo utilizada no Japão e na Coreia do Norte e do Sul, expandindo-se, posteriormente, por toda a Ásia. A acupuntura alcançou o mundo ocidental, a partir da década de 1970. A acupuntura tem como objetivo alinhar e direcionar a energia, por meio da incitação de pontos de acupuntura com agulhas, laser ou acupressão, estimulando os neurotransmissores do sistema nervoso central ${ }^{(8)}$.

A inserção das Políticas Integrativas e Complementares no SUS (PICS), consequentemente, incita discussões ético-legais no que diz respeito ao exercício profissional na aplicação dessas práticas no sistema de saúde, especialmente quando circunda questões de natureza ética do cuidado, que envolvem a aplicação de PICS por profissionais de saúde, em particular, por enfermeiros ${ }^{(9)}$.

Em 2006, o Ministério da Saúde criou o Política de Práticas Integrativas e Complementares, de caráter multiprofissional, possibilitando também que o enfermeiro aplique a Acupuntura como tratamento para diversos 
agravos de saúde, criando Núcleos de Apoio à Saúde da Família, de forma segura, eficaz e autônoma ${ }^{(10)}$.

O tempo recomendável à realização de uma sessão de acupuntura é em torno de 40 minutos, contida essa não apenas como a inserção e manipulação das agulhas, mas também a reavaliação do paciente, a localização dos pontos a serem aplicadas as agulhas e o seu reposicionamento, sempre que necessário. 0 tempo de permanência das agulhas é variável, conforme o quadro clínico e os objetivos do tratamento, sendo, em média, de 20 minutos. Um atendimento de excelência, que proporciona uma escuta diferenciada e qualificada leva a sessão de acupuntura à uma boa prática aplicada na enfermagem.

O termo "boas práticas" é empregado para o cuidado em saúde, desde 1990, a partir da discussão sobre as ferramentas de gestão da qualidade nas instituições de saúde, promovida pela Organização Panamericana de Saúde OPAS $^{(11)}$.

A "Boa Prática" ou "Melhor Prática" ou "Prática Avançada" ou "Práticas Baseadas em Evidências" é, usualmente, definida pela Organização Mundial da Saúde (OMS) como uma técnica ou metodologia que, por meio da experiência e da investigação, possui uma credibilidade comprovada para carrear um determinado resultado. No âmbito dos programas e serviços de saúde, a definição prática de "Melhores Práticas" equivale ao conhecimento sobre aquilo que funciona em situações e contextos específicos, sem a utilização desmesurada de recursos para atingir os resultados desejados, e que pode ser utilizado para desenvolver e implementar soluções adaptadas a problemas de saúde semelhantes noutras situações ou contextos. Resumidamente, boas práticas é um conjunto de compromissos para se fazer uso do corpo de conhecimentos, ao nosso dispor, garantindo êxito em nossas intervenções ${ }^{(12)}$.

O enfermeiro de prática avançada tem reconhecimento social em países como Estados Unidos da América, Canadá e Reino Unido, que desfrutam de estrutura regulatória da profissão bem estabelecida, o que ainda não se consolidou no Brasil e nem em outros países da América Latina, inexistindo diretrizes relativas à capacitação educacional, prática e regulamentação do exercício profissional. Diante de tal cenário e com a importância estratégica dos recursos humanos em saúde para o alcance da meta da Cobertura Universal de Saúde e do Acesso Universal de Saúde, a OPAS concebeu o plano de trabalho para a expansão e profissionalização da Enfermagem de Prática Avançada, intensificando a busca por uma identidade comum entre enfermeiros latinoamericanos e a união da cultura de enfermagem para embasamento à padronização do papel do enfermeiro de prática avançada ${ }^{(13)}$.

A ausência de publicações referentes aos avanços, às limitações da enfermeira acupunturista, em junção ao interesse, por pesquisar essa temática foi despertado, ao longo da vivência profissional desta pesquisadora, especialista em Acupuntura. Neste estudo, objetivou-se conhecer os avanços na atuação das enfermeiras na prática da acupuntura em Santa Catarina, no período de 1997 a 2015.

\section{MÉTODO}

O método de pesquisa utilizado, neste estudo, foi o qualitativo na abordagem históricoSocial, que se define como uma abordagem sistemática, por meio de coleta, organização e avaliação crítica de dados que têm relação com ocorrências do passado, que compreendem o estudo dos grupos humanos, no seu espaço temporal e se preocupam em discutir os variados aspectos do cotidiano das diferentes classes e grupos sociais $^{(14)}$.

O recorte histórico é de 1997 a 2015, justificando seu início pelo reconhecimento do exercício da acupuntura, para a profissional enfermeira, respaldada na Resolução COFEN ( $n$ 197, 1997). E, o recorte final concentra-se em 2015, com a ampliação da Política Nacional de Práticas Integrativas e Complementares ${ }^{(15)}$.

A técnica metodológica utilizada foi a História Oral Temática, como fonte primária, que trata de um objeto específico vivido pelo indivíduo. O método de análise utilizado para compor este estudo foi o da análise temática, onde a fala dos entrevistados foi situada no seu contexto para melhor compreensão(16).

Participaram desta pesquisa 19 enfermeiras especialistas em acupuntura e um (1) enfermeiro com a mesma formação, ou seja, 20 profissionais de enfermagem. Para a seleção dos entrevistados, foi realizado contato com o Conselho Regional de Enfermagem, seção Santa Catarina (COREN/SC) para identificação das profissionais Enfermeiras Especialistas em Acupuntura. Além disso, buscou-se as Escolas de 
Formação em Acupuntura, uma localizada em Florianópolis, nomeada Escola X e Escola Y, com sede no Paraná e subsedes em Santa Catarina, para complementar a busca. A escolha das escolas justifica-se, a primeira por ser a escola referência no estado e, a segunda por ter subsedes em SC e ter sido a escola de formação da pesquisadora. Para um maior alcance de participantes, foi utilizado o método snowball. Como critério de inclusão, utilizamos profissionais que adquiriram o título de especialista, entre 1997 até 2015, com atuação na área no período do recorte histórico. Os participantes da pesquisa foram identificados com o nome de pontos de acupuntura. Segue abaixo a lista dos participantes, com titulação, área de formação, idade e cidade:

1. Tài Yan, Mestre e Enfermeira - 70 anos, Blumenau

2. Dà Líng, Doutora e Enfermeira, 63 anos, Florianópolis

3. Shèn Mén, Enfermeira Especialista, 62 anos, Florianópolis

4. Tài Chong, Enfermeira Especialista, 60 anos, Tubarão

5. Tài Bái, Mestre e Enfermeira, 55 anos, Florianópolis

6. Tài Xi, Mestre e Enfermeira, 54 anos, Florianópolis

7. Zhong Fu, Enfermeira e Especialista, 54 anos, Joinville

8. Jù Què, Doutoranda e Enfermeira, 42 anos, Florianópolis

9. Zhang Mén, Mestre e Enfermeiro, 42 anos, Florianópolis

10. Jing Mén, Mestre e Enfermeira, 41 anos, Tubarão

11. Yún Mén, Enfermeira Especialista, 39 anos, São José

12. Shen Tang, Mestre e Enfermeira, 37 anos, Florianópolis

13. Shen Feng, Enfermeira Especialista, 37 anos, Içara

14. Shen Cang, Doutora, Enfermeira e Naturóloga, 34 anos, Florianópolis

15. Ben Shen, Enfermeira Especialista, 34 anos, Florianópolis

16. Shen Que, Enfermeira Especialista, 33 anos, Chapecó

17. Shen Dao, Enfermeira Especialista, 32 anos, Florianópolis

18. Shen Ting, Enfermeira Especialista, 32 anos, Santa Terezinha

19. Ting Hui, Doutora e Enfermeira, 31 anos,

\section{Florianópolis}

20. Er Men, Enfermeira Especialista, 31 anos, Tubarão

Para garantir os cuidados éticos e a validade dos documentos coletados, a coleta de dados seguiu um rigoroso conjunto de procedimentos para a constituição das narrativas, procedimentos estes que podem ser colocados nas seguintes etapas: aplicação do instrumento de entrevista (composto de 16 perguntas); gravação das entrevistas; transcrição, validação e análise $\mathrm{e}^{(14)}$.

Este estudo foi aprovado pelo Comitê de Ética em Pesquisa com seres humanos da UFSC, em 26 de março de 2018, parecer n. CAAE 2.562.782. Os profissionais foram contactados por email, sendo realizado o primeiro contato de aproximação. O segundo momento tratou do envio dos convites de participação na pesquisa, TCLE e termo de cessão, via email. Como os trâmites até chegar aos profissionais foram longos e os prazos foram se estreitando, por conta disso foram realizadas 10 entrevistas via áudio de WhastApp e 10 presenciais. As entrevistas presenciais foram em locais agendados, no período do final de março a maio de 2018, com tempo médio de uma (1) hora por entrevista. Todas as entrevistas foram gravadas utilizando-se um gravador digital, foram transcritas, validadas por meio do termo de cessão de entrevista. Os relatos foram analisados, comparados e agrupados por similaridade de conteúdo, como uma maneira de organizar a discussão. Da análise temática, de acordo com Minayo, foram elencadas duas categorias, que são apresentadas como resultado desta pesquisa.

Este estudo encontra-se alicerçado na sociologia das profissões de Eliot Freidson, o qual apresenta três fatores, que descrevem o tipo ideal de profissionalização: autonomia, expertise e credenciais de qualidade sobre o seu respectivo trabalho ${ }^{(17)}$.

\section{RESULTADOS E DISCUSSÃO}

Este estudo apresenta seus resultados e discussão nas categorias criadas para facilitar sua descrição. Na categoria 1. Avanços e Desafios da Enfermeira Acupunturista - um (1) participante no quesito avanços falou sobre as limitações que impedem o avanço; sete (7) participantes expuseram suas opiniões sobre os avanços da classe; (11) participantes falaram sobre o desafio profissional, a OMS, as PNPICS, COFEN e CORENS; 
um (1) participante referiu a inexistência de avanços. Na categoria 2. Boas Práticas da Enfermeira na acupuntura - três (3) participantes associaram a boa prática à escuta, cinco (5) participantes reuniram boas práticas no atendimento de acupuntura com a aplicação de outras técnicas, dois (2) participantes falaram que as boas práticas no seu atendimento estão diretamente ligadas à biossegurança do paciente, seis (6) participantes descrevem a acupuntura como uma boa prática dentro do seu processo de cuidado, um (1) participante não identifica uma boa prática no seu atendimento.

\section{Avanços e Desafios da Enfermeira Acupunturista}

Ainda que a pequenos passos, a história das enfermeiras acupunturistas vêm evoluindo. Limitações existem e estas impedem que o crescimento aconteça efetivamente. Os Desafios profissionais e as insatisfações com as entidades de interesse público estão presentes no cotidiano profissional. As falas que seguem caracterizam limitações ao avanço das práticas da acupuntura por Enfermeiras:

"Eu não cheguei acompanhar desde o início da resolução do COFEN que permite que o enfermeiro praticasse acupuntura, mas o que eu percebo aqui agora com mais essa resolução que firma a possibilidade do enfermeiro atender em seu próprio consultório e acredito que existem ainda mais possibilidades do enfermeiro atuar na consulta de enfermagem a acupuntura. Prova disso é que já é possível observar enfermeiros que atuam exclusivamente nessa área com consultórios às vezes em parceria com outros profissionais em consultórios multiprofissionais e desenvolvendo todas as ações pertinentes ao enfermeiro. As pessoas deixam de ver o serviço de enfermagem como um serviço relacionado especificamente às questões da caridade, da ajuda ao próximo, como aconteceu no início da sua história enquanto profissão e passam a saber que a enfermagem possui conhecimentos técnicos científicos capazes de contribuir para as questões de saúde e qualidade de vida das pessoas" (Shen Que).

"Eu penso que nós poderíamos enquanto profissionais envolvidos na medicina tradicional chinesa ter uma produção científica maior. Há um avanço bem limitado principalmente não só pela enfermagem mas de um modo geral profissionais que desenvolvem a terapia chinesa ainda tem uma grande dificuldade em documentar e realizar pesquisas sobre o seu trabalho sobre o seu processo de atenção à saúde. Seria um passo importante para o avanço na área para os profissionais que atuam e para a área de medicina tradicional chinesa na saúde no mundo ocidental em geral" (Dà Líng).

"Não percebi muito avanço, existem algumas áreas que eu acho que caminharam mais fácil, mais rápido, como fisioterapia, mas da enfermagem vejo pouco avanço" (Ben Shen).

"Eu vejo que ainda caminha devagar por causa dessas questões do médico estar retendo para ele, as facilidades de um convênio de saúde. A gente acaba perdendo cliente, para eles dessa forma, pelas facilidades que o usuário tem. Eu acho que a gente tem de continuar tentando, por que assim cada vez mais as pessoas estão aderindo às práticas integrativas" (Zhong Fu).

As falas a seguir caracterizam os avanços da classe:

"Eu acho que teve uma grande evolução no percurso, por conta da questão dos profissionais que estão se especializando, divulgando o serviço, e acredito que em nivel de SUS pela política nacional de práticas integrativas e complementares. No ano passado práticas foram ampliadas, tendo uma grande divulgação no jornal. Teve as olimpíadas, apareceram as ventosas na mídia, agora todo mundo sabe o que é ventosaterapia" (Jing Mén).

"Eu acho que a Enfermagem está avançando, porque ela está regularizada, então enfermeiro acupunturista hoje está regularizado e pode abrir um consultório e ele pode atender. $\mathrm{Eu}$ acho que isso é um avanço para a enfermagem. Eu acho que o próprio conselho, nosso COREN atuou de forma decisiva, para que isso ocorresse" (Tài Xi).

"Eu conheci a associação brasileira de enfermeiros acupunturistas e de práticas integrativas. A ABENAH. Fui em um evento e a presidente da ABENAH estava lá. , a presidente, justamente foi pra esse evento pra tentar fazer a divulgação do trabalho da associação brasileira de acupuntura. Se não nos fortalecermos perderemos espaço. A primeira história do ato médico muito enfermeiro caiu fora porque achou que não ia ter pulso suficiente para poder bancar a profissão. Acho que esses avanços ajudam a fortalecer e mostrar que a gente tem força que a gente faz um trabalho bacana e que a gente pode sim fazer a diferença dentro inclusive de um SUS, com acupuntura, dentro de um hospital com acupuntura" (Jù Què). 
"Vejo uma evolução bem grande. A primeiras enfermeiras que fizeram curso de acupuntura mais ou menos trabalhavam na clandestinidade, não eram reconhecidas. Como é que você, enfermeira faz acupuntura? Sempre eu era questionada dentro do meu estúdio. Eu respondia: eu não faço acupuntura médica, ou de enfermagem, eu faço acupuntura dentro da medicina tradicional chinesa. Então eu acho que isso é um grande avanço para a enfermagem e para as enfermeiras em geral. Essa liberdade que a enfermeira tem como profissional autônomo e você pode sim procurar enfermeira para fazer uma consulta de pré-natal ou procurar uma enfermeira para fazer uma consulta de acupuntura. Os profissionais docentes que trabalham em Universidades formando cada vez mais Mestres e Doutores enfermeiros e profissionais liberais" (Shén Mén).

As atitudes devem partir também de nós profissionais para que mudanças aconteçam e consequentemente conquistas ocorram no âmbito da expertise profissional escolhida/adquirida.

Um dos grandes avanços do exercício da acupuntura foi que antigamente ela era exercida por pessoas que não apresentavam formação na área da saúde ou mesmo formação universitária. Cabe destacar o avanço no atendimento da enfermeira acupunturista realizado uma vez por semana em uma Unidade Básica de Saúde, em Florianópolis, na capital de Santa Catarina, com agenda direcionada exclusivamente a consulta de acupuntura, embora seja recebendo remuneração de enfermeira da UBS.

Os avanços ocorreram e os órgãos representativos, os COFEN e COREN deram e continuam dando força para execução dos serviços prestados dentro do país. Eles consentem uma reflexão e execução bem mais ampla, retratando como as enfermeiras acupunturistas se apresentam hoje no Brasil com seus avanços e conquistas de seus direitos trabalhistas e sociais. Para que mais avanços ocorram as profissionais acupunturistas precisam buscar e implantar a prática de forma multiprofissional, técnica, competente, democrática, compartilhada e ética, com intuito de beneficiar a saúde das pessoas que usufruirão da técnica ou terapêutica, como assim podemos chamar.

Os desafios identificados neste estudo nos levam a pensar que a enfermagem não deve ficar apenas à margem da discussão sobre a regulamentação do emprego de Práticas Integrativas e Complementares pelo SUS, como deve também refletir e reivindicar sobre as possibilidades de legitimação destas práticas no âmbito do cuidado de enfermagem. A visão holística do enfermeiro agregada às práticas complementares executa um papel fundamental na sua aplicabilidade ${ }^{(9)}$.

As Práticas Integrativas e Complementares (PICS) desempenham um considerável papel na saúde das pessoas, atuando especificamente na promoção, prevenção e reabilitação. E os enfermeiros são os profissionais de destaque na implementação e utilização das PICS, possuindo respaldo legal, podendo atuar em serviços públicos e privados. Os enfermeiros se mostram atuantes em pesquisas dentro das universidades, na prática clínica nas unidades básicas de saúde e nas instituições privadas, contribuindo para a divulgação de conhecimentos embasados cientificamente e para a disseminação das PICS à comunidade, mesmo que ainda de forma lenta. As PICS oportunizam autonomia, por meio de uma ação terapêutica eficiente aos enfermeiros e estes devam reconhecer a oportunidade que se expande para a profissão, com as terapias integrativas dentro das PICS. Ainda é pequeno o número de profissionais que atuam com essas terapias, no entanto, ainda muito precisa avançar. Um longo caminho precisa ser percorrido no sentido de fortalecer a utilização das PICS pelos enfermeiros no SUS encontrando nestas terapias uma perspectiva de intervenção em prol da sociedade ${ }^{(18)}$.

\section{Boas Práticas da Enfermeira na Acupuntura}

Muito se fala em como devemos nos expressar para garantir uma ótima comunicação. Porém, a comunicação é uma via de mão dupla. Saber ouvir é tão importante quanto saber falar. As Boas Práticas podem estar presentes durante um atendimento entre outras técnicas. Assim como a acupuntura como especialidade dentro da enfermagem pode ser vista como uma boa prática durante $\mathrm{o}$ atendimento, enfermeiracliente/paciente. Os resultados a seguir detalham falas sobre boas práticas:

"Durante a consulta eu faço aferição de PA, vejo a saturação, a frequência cardíaca, faço HGT, vejo os exames também registro eles, além de fazer a prática de olhar a língua, ganhamos credibilidade quando a gente vincula a medicina ocidental junto com a medicina chinesa porque se a gente for falar só de vento frio, das filosofias 
da medicina chinesa parece que ninguém entende. Eu já vi outros colegas que atendem e há o paciente está com dor no ombro, faz analgesia e deu. Eu acho que é o meu diferencial é a escuta qualificada" (Er Men).

"Junto com acupuntura sempre oriento alguns tipos de alimentos que poderiam ajudar e converso bastante com os pacientes. Escuto, porque eu vejo que muitos estão naquele momento ali também para conversar. Eu estou sempre pronta para conversar, para ouvir eles" (Shen Ting).

"Eu usava muito o hidoraku que não são todos que usam. Eu fazia consultas demoradas de duas horas. Reflexologia podal dependendo de cada caso, claro, não utilizava todas as técnicas, mas o que desse para complementar o atendimento" (Ben Shen).

"Eu acho que é esse atendimento individualizado da pessoa, é, o fato de estar associando outras práticas em conjunto, até porque eu sou naturóloga então eu domino outras práticas e associo com floral, associo com aromaterapia, às vezes, enquanto ele tá ali quietinho, quando faço acupressão, em seguida aplicando reiki. Esse é o meu diferencial, por isso eu acho difícil eu falar eu sou enfermeira. Eu sou naturóloga, eu faço tudo junto, e esse olhar integrado, tentando ver do psíquico ao social e o ambiental" (Shen Cang).

"Meu mestrado foi um diferencial, ter trabalhado com pessoas com lesão medular. Pelo que eu pesquisei principalmente trabalhando a sexualidade na pessoa com lesão medular não encontrei nenhum artigo publicado, então minha contribuição, meu diferencial seria esse. A escuta diferenciada também é uma boa prática" (Shen Tang).

As (Boas) Práticas da Enfermagem, em qualquer âmbito de ação, devem conter pressupostos ou princípios. É preciso entender que o processo saúde-doença está de modo direto agregado ao potencial de desgaste e de fortalecimento dos grupos sociais, e que, ao lado de valores e contra- -valores derivados dos processos de reprodução social, convertem-se no processo bio-psíquico-social do indivíduo ${ }^{(19)}$.

Os enfermeiros são profissionais autônomos e, no âmbito dessa autonomia, há a pertinência desta prática. $O$ enfermeiro enquanto membro de uma equipe não trabalha nem toma decisões sozinho Já a enfermeira apropriada da sua expertise, neste caso, a acupuntura apresenta autonomia e toma as decisões independentes e de acordo com seus conhecimentos. A autonomia profissional é uma especificidade que confere poder a profissão, atingindo o seu status quando executa o seu fazer sob a proteção da sociedade e do controle estatal ${ }^{(17)}$.

Os fundamentos da profissão de enfermagem são os de cuidar, promover e prevenir. Os da acupuntura são os cinco elementos que regem o corpo humano e a natureza a qual este corpo encontra-se inserido, resultando no cuidado, promoção e prevenção, ou seja, a enfermagem e a acupuntura unem-se em prol do bem-estar e da saúde.

Para Freidson, o progresso da expertise é indispenśavel para demonstrar a autoridade em coordenar a divisão de trabalho de uma profissão ${ }^{(17)}$. A inserção da enfermagem e sua identidade dar-se-á por meio da construção de uma identidade política, ética, científica que expresse a responsabilidade e a autonomia deste profissional A busca pelo conhecimento e a busca pelo poder profissional estão lado a lado para Freidson ${ }^{(20)}$.

Faz-se necessário mencionar ainda que a introdução da acupuntura no Brasil, assim como em outros países, esteve intimamente relacionada ao processo de migração de profissionais do Oriente com domínio desta prática, os verdadeiros responsáveis pela sua inserção no país ${ }^{(21)}$.

A representatividade do sistema COFEN e dos COREN nos remete ao terceiro fator de profissionalização de Freidson, que é o credencialismo, o conjunto de regras e regulamentos formais corporificados em Leis ou a regulamentos e resoluções vinculados a instituições políticas, associações profissionais e organizações educacionais. O Credencialismo posiciona-se com o amparo político e judicial para que o profissional exerça sua atividade, reitera-se por meio de um título ou credencial, predizendo desta forma, a regulamentação profissional por lei e atuação dos Conselhos Profissionais ${ }^{(22)}$.

Os três fatores que narram o tipo ideal de profissionalização: autonomia, expertise e credenciais qualificacionais vão ao encontro dos avanços da enfermeira acupunturista ${ }^{(20)}$. As (Boas) práticas aplicadas durante o atendimento, sendo estas os frutos dos avanços profissionais da enfermeira acupunturista. Os diagnósticos de enfermagem podem sofrer intervenções por meio da acupuntura, podendo tanto ser aplicada por enfermeiros especialistas quanto indicada 
por outros não especialistas, mas que reconheçam na acupuntura uma possibilidade interventiva.

\section{CONCLUSÃO}

Ao analisarmos desafios vem a tona a fragilidade do pouco reconhecimento de enfermeiras na área da acupuntura. As enfermeiras acupunturistas devem reivindicar sobre as possibilidades de legitimação da acupuntura no âmbito do cuidado de enfermagem dentro do Sistema ùnico de Saúde.

A maior conquista dessa categoria é subjetiva e está relacionada ao encontro com o outro no ato de cuidar, sendo esse, o mais compensador, legitimador de ações humanizadas, de espírito afetivo e nobre. Um grande avanço é que a sociedade que reconhece que as ações da enfermeira acupunturista requer estudo, pesquisa, dedicação e habilidades para serem exercidas de maneira ímpar.

A enfermagem, por ser uma ciência de natureza humanística, encontra na acupuntura uma nova forma e também uma "Boa Prática" para melhor atender seus clientes, com ênfase na promoção da saúde e na prevenção de doenças. As boas práticas aliadas a Sistematização da Assistência em Enfermagem consolidam a prática baseada em evidências e garantem a expertise, oferecendo a autonomia as profissionais e promovendo a segurança de pacientes e comodidade sob a responsabilidade das credenciais profissionais.

Frente ao exposto e apesar da Enfermagem hoje se constituir uma das maiores e mais importantes profissões na área da Saúde no Brasil e a acupuntura se constituir também uma especialização dentro da enfermagem, ela vem ganhando destaque dentro da profissão na área da Saúde a nível de Brasil, mas mesmo assim observamos ainda que temos muito e devemos na mesma proporção avançar.

\section{REFERÊNCIAS}

1- Contatore OA, Malfitano APS, Barros NF. Care process in the health field: ontology, hermeneutics and teleology. Interface 2017;21(62):553-63. DOI: $10.1590 / 1807-$ 57622016.0616

2- Queirós PJP, Fonseca EPAM, Mariz MAD, Chaves MCRF, Gómez CS. Significados atribuídos ao conceito de cuidar. Rev Enf Ref. 2016;4(10):85-94. DOI: 10.12707/RIV16022

3- Hicks A, Hicks J, Mole P. Acupuntura constitucional dos cinco elementos. São Paulo: Rocca; 2007.

4- Brasil, Conselho Federal de Enfermagem. Resolução no 197/1997. Estabelece e reconhece as Terapias Alternativas como especialidade e/ou qualificação do profissional de Enfermagem. Rio de Janeiro: COFEN; 1997.

5- Brasil, Conselho Federal de Enfermagem. Resolução no 326/2008. Regulamenta no Sistema COFEN/CORENs a atividade de acupuntura e dispõe sobre o registro da especialidade. Rio de Janeiro: COFEN; 2008.

6- Associação Brasileira de Enfermeiros Acupunturistas e Enfermeiros de Práticas Integrativas (ABENAH). [citado em 30 jan 2019]. Available in: http://abenanacional.org/2018

7- Sousa LA, Barros NFd, Pigari JO, Braghetto GT, Karpiuck LB, Pereira MJB. Acupuncture in Brazil's Unified Health System - an analysis based on different health management tools. . Rev Ciênc Saúde Coletiva. 2017 ;22(1):301-10. DOI: 10.1590/1413-81232017221.10342015

8- Goyatá SLT, Avelino CCV, Santos SVM, Souza Junior DI, Gurgel MDSL, Terra FS. Effects from acupuncture in treating anxiety: integrative review. Rev Bras Enferm. 2016;69(3):602-9. DOI: 10.1590/0034-7167.2016690325i

9- Magalhães MGM, Alvim NAT. Complementary and integrative therapies in nursing care: An ethical focus. Esc Anna Nery 2013;17(4):646-53. DOI: 10.5935/1414-8145.20130007

10- Telesi Júnior, E. Práticas integrativas e complementares em saúde, uma nova eficácia para o SUS. Rev Estud Av. 2016;30(86):99-112. DOI: 10.1590/S0103-40142016.00100007

11- Lopes LA, Bernardino E, Crozeta K, Guimarães PRB. Good practices in collecting umbilical cord and placental blood. Rev LatinoAm Enfermagem 2016;24:e2770. DOI: 10.1590/1518-8345.0781.2770

12- Organização Mundial da Saúde (OMS). Registo no catálogo de publicações da Biblioteca AFRO: guia para a documentação e partilha das "Melhores Práticas" em programas de saúde. África: OMS; 2008.

13- Scochi CGS, Gelbcke FL, Ferreira MA, Alvarez ÂM. Professional Master's Degree: potential contribution to Advanced Practice Nursing. Rev Bras Enferm. 2015;68(6):1186-9. DOI: $\underline{10.1590 / 0034-7167.2015680626 \mathrm{i}}$ 
14- Padilha MI, Bellaguarda MLR, Nelson S, Maia ARC, Costa R. The Use Of Sources In Historical Research. Texto-Contexto Enferm. 2017;26(4):e2760017. DOI: 10.1590/010407072017002760017

15- Brasil, Ministério da Saúde. Política nacional de práticas integrativas e complementares no SUS: Atitude de ampliação de acesso. 2a ed. Brasília: Ministério da Saúde; 2015.

16- Minayo MCS, Holanda F. O desafio do conhecimento: Pesquisa qualitativa em saúde. 14a ed. São Paulo: Hucitec; 2014.

17- Freidson E. Profissão médica: Um estudo de sociologia do conhecimento aplicado. São Paulo: UNESP; 2009.

18- Azevedo C, Moura CC, Corrêa HP, Mata LRF, Chaves ÉCL, Chianca TCM. Complementary and integrative therapies in the scope of nursing: legal aspects and academic-assistance panorama. Rev Esc Anna Nery Enferm. 2019;23(2):e20180389. DOI: 10.1590/2177-9465ean-2018-0389

19- Egry EY, Fonseca RMGS, Oliveira MAC, Bertolozzi MR. Nursing in Collective Health: reinterpretation of objective reality by the praxis action. Rev Bras Enferm. 2018;71(supl 1):710-5. DOI: 10.1590/0034-7167-2017-0677

20- 20-Freidson E. Renascimento do profissionalismo: Teoria, profecia e política. São Paulo: Edusp; 1988.

21- Rocha SP, Benedetto MAC, Fernandez FHB, Gallian DMC. A trajetória da introdução e regulamentação da acupuntura no Brasil: Memórias de desafios e lutas. Ciênc Saúde Coletiva. 2015;20(1):155-64. DOI: 10.1590/141381232014201.18902013

22- Bellaguarda MLR, Padilha MI, Pereira NAF, Pires D, Peres MAA. Reflexão sobre a legitimidade da autonomia da enfermagem no campo das profissões de saúde à luz das ideias de Eliot Freidson. Esc Anna Nery 2013;17(2):369-74. DOI: $10.1590 /$ S1414-81452013000200023

\section{Nota:}

Agradecemos à CAPES e ao CNPQ pelo apoio no desenvolvimento da pesquisa e bolsa de estudo para os Cursos de Mestrado e Doutorado.

Este artigo é parte da dissertação de mestrado da autor correspondente, intitulada: "Historicidade da Prática da Acupuntura por Enfermeiras em Santa Catarina no Período de 1997 A 2015" apresentada ao Programa de Pós Graduação em Enfermagem da Universidade Federal de Santa Catarina no dia 17 de dezembro de 2018.
Received in: $17 / 02 / 2020$

Approved in: 03/06/2020

Mailing address:

Ana Paula Senna Bousfield

R. Delfino Conti, s/n. CCS UFSC - Campus Trindade Florianópolis SC.

paula.bousfield@gmail.com 